\section{No. 4679 July 4,1959 \\ Electron Microscopical Structure of the Wall of Small Blood Vessels in Human Multiform Glioblastoma}

WHILE using the electron microseope to study the fine structure of the small vessels surrounding central necroses, typical of multiform glioblastoma, endothelial proliferation and the presence of processes arising from the 'basement membrane' were observed. The fine structure of the latter differed essentially from that in normal vessels. The present observations are in accordance with earlier light microscopical observations ${ }^{1,2}$

The material used consisted of four cases of multiform glioblastoma and three cases yielding tissue of origin, namely, normal brain tissue. Specimens were fixed in osmic acid, and embedded in methacrylate. Ultra-thin sections were examined with a Philips $E M 100 B$ electron microscope.

The structure of the basement membrane of the blood vessels in multiform glioblastomas (Fig. 3) differed from normal basement membrane (Fig. 1) in that it exhibited considerable variations in thickness, which was in the range $300-2500 \mathrm{~A}$., and had a relatively irregular outline forming numerous branches extending deeply into and between the endothelial cells. The laminar structure of the basement membrane of the capillaries was largely dissolved. The basement membrane of the venules and arterioles showed similar alterations. In the wall of the latter the collagen fibres appeared to be detached from the elastic framework, so that the whole elastica interna exhibited an extremely loose structure; at certain places the basement membrane was very thin and incomplete and the endothelium consequently stretched (Fig. 4).

The blood capillaries in multiform ghioblastome were generally characterized by a high degree of endothelial proliferation. The size of the nuclei and the amount of cytoplasm were increased (Fig. 2), as compared with capillaries of normal brain tissue. Furthermore, in some instances the lumen was completely filled with endothelial cells. The endothelial layers appeared to be continuous. Frequently the mitochondria in the endothelium were so densely packed that they were separated from each other only by thin membranes. The presence of processes with a layered strueture in the endothelium and in part also between the endothelial cells was particularly striking where marked proliferation of the endothelial cells occurred, but less conspicuous elsewhere. The present findings seem to indicate that the intraendothelial collagen does not originate in the endothelial cells, but in the external layers : the basement membrane or the elastica interna. The cells in the endothelial proliferation appeared to have lost their polarity. When the lumen became markedly narrow or totally occluded, the augmented pressure in the blood-vessel may have resulted in dilatation of the vessel on the proximal side of the occlusion, that is to say, on the side of the inflowing blood-stream, where the basement membrane or the elastica interna already was defective. This appears to be the mechanism in the development of irregularities and especially of the numerous small aneurysms characteristic of multiform glioblastomas.

Some of the cells in the pericapillary region were in intimate contact with the capillary walls through organelles resembling the Golgi apparatus. In these sites the basement membrane showed a more reticular

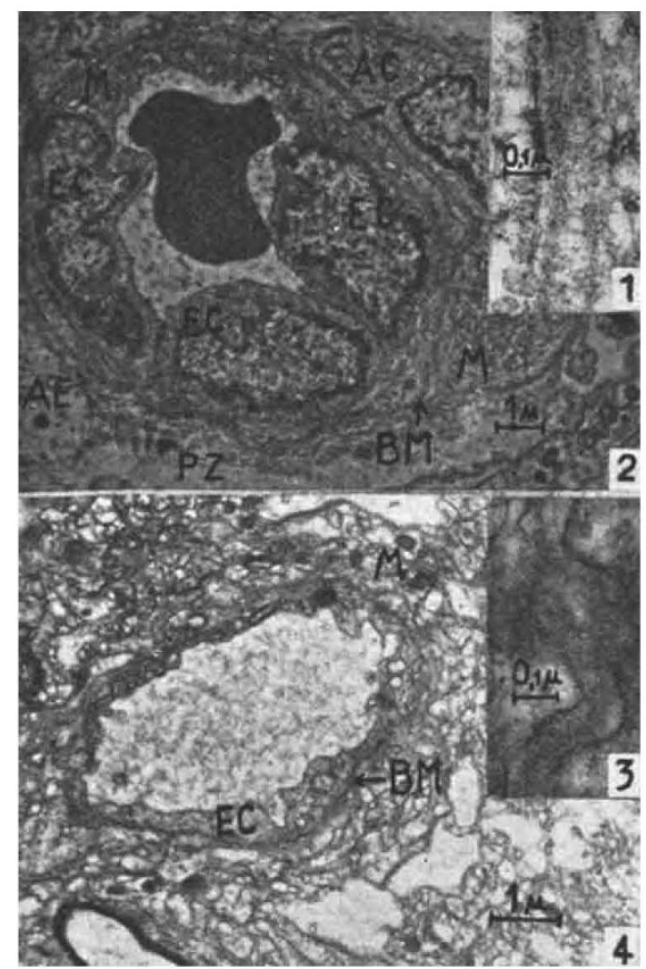

Fig. 1. Basement membrane ( $B M$ ) of blood vessel in normal brain tissue. A longitudinal layer in the margins and a radiaily organized lamellar structure in the middle. $\times 45,000$

Fig. 2. Hypertrophy and proliferation of endothelial cells $(E C)$ in capillary wall of a glioblastoma. Pericapillary zone $\left(F^{\prime} Z\right)$ Left : mitochondria $(M)$ at the margin of an astrocytic end-foot $(A E)$. Right: an 'additional cell' $(A C) . \times 5,000$ Fig. 3. $B M$ of a capillary in a glioblastoma. Irregular outline
and varying diameters. The structure is more vesicular than lamellar (cf. Fig. 1) $\times 45,000$

Fig. 4. Capillary with an extremely thin and incompletely developed $B$ M. $\times 7,400$

structure. In close vicinity to the capillaries, between them and the necroses, numerous erythrocytes and lymphocytes with granular cytoplasm were seen, a finding often reported in conventional microscopic investigations of glioblastomas. In contrast to the capillaries in normal brain tissue, a large part of the capillaries of glioblastomas had a pericapillary zone of lower density.

The electron micrographs of normal vessels controls) confirmed previous observations. In addition, the present findings of the structure of the normal basement membrane were corroborated by polarization microscopic observations ${ }^{3}$.

Further results will be published later in another connexion, with special reference to the fine structure of the wall of the small vessels in othor gliomas and in metastatic tumours.

This work was supported by a research grant from the University of Helsinki.

\section{S. H. M. NystRö̀}

Institute for Electron Microscopy,

University of Helsinki. March 22.

I Deery, E. M., Bull. Neurol. Inst. N.Y., 2, 157 (1932),

"Zuiich, K. J., and Christensen, E., "Handbuch der Neurochirurgie", 3 (Springer Verlag, Berlin, 1936)

${ }^{3}$ Niessing, K., and Rollhäuser, H., Z. Zellforsch., 38, 431 (1954) 